\title{
An In Vitro Model for Evaluating Bacterial Leakage Through Endodontic Repair Materials
}

\author{
P. Sreekumar ${ }^{1}$, Jolly Mary Varughese ${ }^{2}$, N.O. Varghese ${ }^{3}$, Manoj Komath ${ }^{4}$ and \\ H.K. Varma ${ }^{4}$ \\ ${ }^{1}$ Department of Conservative Dentistry and Endodontics, Sri Sankara Dental College, India. \\ 2 Government Dental college, Trivandrum, INDIA \\ 3 PMS College of Dental Science and Research, Trivandrum, INDIA \\ ${ }^{4}$ Biomedical Technology Wing, Sree Chitra Tirunal Institute for Medical Sciences and Technology,
} Trivandrum, INDIA

\begin{abstract}
The leakage of bacteria through endodontic repair materials is a matter of great concern in dentistry, as the success of repair of furcation perforations or root ends lies in the seal provided by the material against bacterial infiltrations. In vitro microleakage techniques (like dye penetration and fluid filtration) are used to assess the sealing ability of repair materials. However, such microleakage results cannot be correlated with migration of microbes in real situations. The quest for obtaining clinically relevant data prompted researches to develop bacterial leakage models. Various custom set ups had been developed for the purpose.

We report a new model for studying bacterial leakage through repair materials, which could be applied universally for teeth of any size and shape. It consists of a cast of the repaired tooth in poly methyl methacrylate (PMMA), incorporated in microbiological test set up. The cast is modified to get a delivery chamber in the upper portion and the lower portion is appropriately sized to allow the repaired part come in contact with the culture medium. The inoculum is delivered to the upper chamber and the leakage of the bacteria is identified through the colony formation in the medium. This model is close to clinical condition and provides reliable and repeatable results. Minimum error parameters, ease of working and reliability are additional features.
\end{abstract}

\section{Introduction}

The leakage of bacteria through endodontic repair materials is a matter of great concern in dentistry. The success of repair of furcation perforations or root ends lies in the seal provided by the material against the oral flora. A bacterial infiltration into the supporting area leads to periodontal breakdown. The sealing ability of the endodontic repair materials is, therefore, an important factor in the clinical success of endodontic procedures ${ }^{1}$. A considerable volume of study is available on this aspect of endodontics, with exhaustive reviews ${ }^{2-5}$.

As there are practical difficulties in assessing the bacterial leakage clinically, in vitro microleakage methods are used for the initial evaluation and comparison of the sealing ability of various repair materials. The concept of microleakage relates to the penetration of oral fluids and microorganisms along the material cavity/wall interface following insertion of a restoration. Microspaces are likely at this interface, caused partly by the contraction of the mass of the material during the setting process and partly by the lack of adherence to the cavity walls. Measuring the microleakage could be used to predict the performance of the material in the oral environment ${ }^{5}$.

A variety of penetration and filtration techniques has been developed to determine the microleakage through dental restorations, like dye penetration, radioisotope tracing, electrochemical migration and fluid filtration ${ }^{3,4,6}$. The dye penetration method is the most favoured among these, because of its simplicity and reproducibility. Despite the popularity, the method has obvious limitations in producing any clinically useful data because of the low molecular sizes of penetrating particles, measurements in a single plane and static experimental conditions ${ }^{7,8}$. The fluid filtration method, in which a liquid medium is pressurized through the repaired area, appears more realistic. However, the results cannot be correlated with the migration of microbes in real situation, as there are several other factors which determine the actual migration, like the availability of nutrients and the anti-microbial property of the material ${ }^{5}$.

Though the in vitro microleakage tests enable to assess the comparative performance of the repair materials, the clinical relevance is doubtful. Microleakage measurements obtained by different in vitro methods, using the same materials gave considerable variation in the values, raising serious concerns about the reliability 4,5 .

The limitations of the in vitro microleakage studies and the quest for obtaining clinically relevant data, prompted the researchers to develop bacterial leakage models ${ }^{8-15}$. The basic idea here is to allow bacteria 
to migrate through the repair material and measure the penetration time using microbiological techniques with repaired teeth, simulating conditions as close as possible to the clinical conditions.

The basic experimental set-up is a two-chamber system enclosing the tooth, with the repaired region separating the chambers. The upper (delivery) chamber holds an inoculum of bacteria and the lower (receiving) chamber carries the culture medium. Any bacteria percolates through the imperfections of the material will enter into the culture medium and get multiplied. The time of the migration is taken as the parameter defining the sealing ability of the material.

Though a considerable number of bacterial leakage studies has been performed in the past few years, no acceptable or standardized model did evolve ${ }^{8-15}$. Custom-made set-ups are used, the simplest being enclosing the tooth in a plastic tube and inserting into a bigger tube with culture medium, after sealing the boundaries. This works reasonably well in the case of single roots, where root-end filling materials are studied. However, in the case of studies related to furcation perforation repair, one has to work with molars. Making a two-chamber system using tubes, in this case, is cumbersome and adds a lot of error parameters.

We report a new model for the bacterial leakage studies, which could be applied universally for teeth of any size and shape, with minimum error parameters. The set up essentially consists of the repaired tooth embedded in acrylic (poly-methylmethacrylate) block compatible in size and shape with the microbiological culture vials. The part lower to the repaired region is cut-off so as to allow the bottom surface of the repair material to be in contact with the culture medium. Access cavity prepared in the upper portion of the block serves as the delivery chamber for the bacterial inoculums. This model is close to clinical conditions and provides reliable and repeatable results.

\section{Materials And Methods}

The model being described here is specifically for the testing of furcation perforation repair, though it could be used for testing root-end sealing also. The method has two parts - preparation of the test block and arranging the microbiological study set-up.

\section{The Test Block :}

There are various steps involved, like cleaning of the tooth, casting in acrylic, sizing the cast according to requirement, access cavity preparation and defect repair. The steps are shown schematically in Figure.1.

Cleaning : Freshly extracted mandibular and maxillary molars with well-formed and divergent roots are selected and stored in normal saline. They are then cleansed of soft debris and calculus using an ultrasonic scaler.

Casting: The casting is done in glass moulds of size $24 \mathrm{~mm}$ outer diameter and $30 \mathrm{~mm}$ height (thin-walled $5 \mathrm{ml}$ capacity pharmaceutical glass vials with low brim thickness). The tooth is kept inside such a glass mould with crown portion facing the bottom. The acrylic is prepared by mixing methacrylate monomer and the initiator, benzoyl peroxide (both of AR quality, Aldrich Chemicals) thoroughly in the prescribed ratio. It is allowed to thicken by keeping warm in a water bath and subsequently transferred carefully in to the vial carrying the tooth. All these processes are to be executed in a chemical fume hood to avoid the exposure to monomer vapour.

The acrylic in the vial is allowed to set overnight in a vacuum oven kept at $50^{\circ} \mathrm{C}$, to facilitate the bubble-free setting of the acrylic. This is followed by cooling to room temperature, which should be done at a slow rate to avoid crack formation. The cast can be released by carefully breaking out the glass mould. An optimized casting process will give a transparent cast, without any bubble or crease.

FIGURE 1

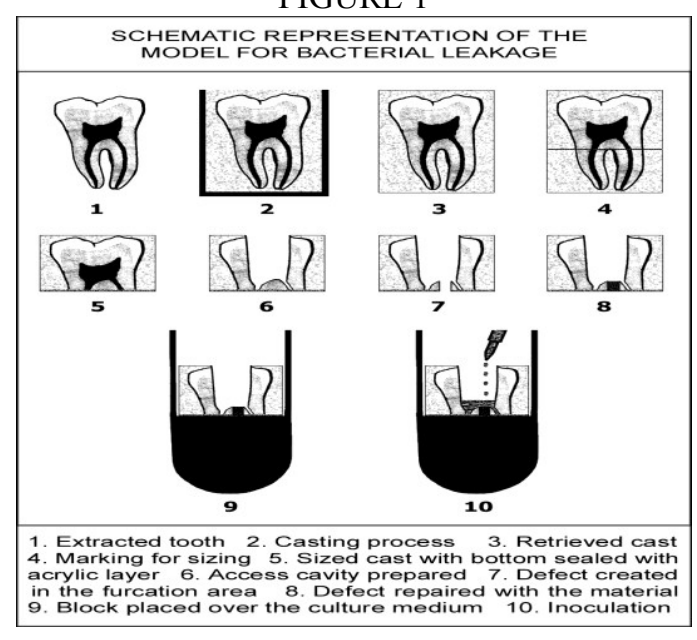


Sizing : The cast is to be sized in height to detach the root portion and expose the furcation area. The furcation level is marked on the periphery of the cast and sectioned using a low speed diamond saw (Isomet, Buehler). The sized part of the cast containing the crown portion, however, cannot be straight away used for testing because the cutting will leave open canals and dentinal tubules at the bottom side. A thin layer $(\sim 0.5 \mathrm{~mm})$ of self-cure clear acrylic was applied over the cut end of the cast to seal the exposed areas of the tooth.

Access Cavity Preparation and Creation of Defect : Access cavity is prepared from the topside of the cast using a round bur (ISO 023). The walls of the preparation are smoothened using a fine taper diamond (ISO 009). The root canal orifice is located using a no:15 K-file (Mallifer, Dentsply). The pulp chamber is cleaned of all pulpal remnants and debris, and the floor of the pulp chamber is perforated using a flat end tapered diamond (ISO 012) till it completely passes through. This results in a perforation of a standard diameter $1.85 \mathrm{~mm}$. However the depth of pulp chamber floor may vary depending upon the thickness of dentin and cementum in that region.

The depth of the perforation (from the floor of the pulp chamber to the bottom of the cast, including the self-cure clear acrylic layer) is measured using a finger plugger with rubber stopper. The finger plugger is inserted through the radicular end of perforation till its tip becomes flush with pulp chamber floor. This length is marked using the rubber stopper and measured using a digital vernier caliper. A uniform depth is preferred in this model, to have a standardized thickness of the repair material inside. The least perforation depth obtainable with mandibular and maxillary molars is observed to be $2.5 \mathrm{~mm}$. Larger perforation depths could be brought down to this value by carefully abrading off the pulpal floor with a flat end burr. This will eliminate errors due to the non-uniform material filling depth.

Sterilization : Sterility of the test samples is a critical factor in a microbiological experiment. The sized cast containing the tooth is sterilized by autoclaving (steam sterilization). From the preliminary investigations, it was found that the acrylic cast withstands the autoclaving conditions, and the tooth acrylic boundary remains intact.

The cast is covered in paper bags and put in the steam sterilizer at $121^{\circ} \mathrm{C}$ and $15 \mathrm{lbs}$ pressure for 20 minutes. After sterilization, the cast is transferred to a biological safety chamber (Class II, with laminar flow of filtered air) in a sterile room for further experiments.

Defect Repair: The defect repair is done at aseptic conditions, in the biological safety chamber. The repair material of interest is mixed as per the manufacturer's specification and filled in the perforation through the access cavity, as done in the actual repair procedure. Wet cotton is used at the bottom side of the cast to simulate the clinical conditions. The repaired cast is kept in the biological safety chamber for 24 hours, in order to allow the proper setting of the material. The coronal and radicular views of the repaired cast are shown in Figure. 2.

FIGURE 2

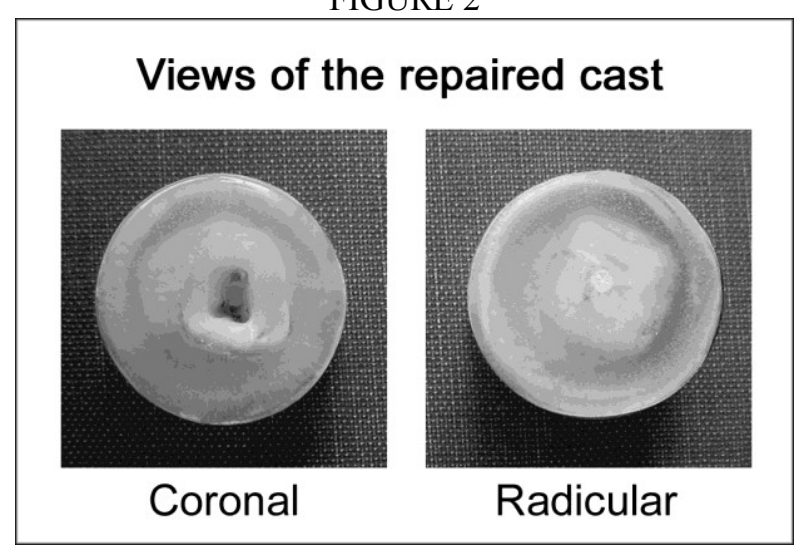

The Microbiological Study Set-up :

The essential part of a bacterial leakage model is the set up for bacterial culture medium. Sterile rimless test tubes $(25 \mathrm{~mm} \times 75 \mathrm{~mm})$ with matching plastic caps are used as the container for the culture medium. The casts made inside a $25 \mathrm{~mm}$ glass mould will precisely slide-in inside the test tube.

An appropriate culture medium (like phenol red broth, brain-heart infusion broth or Hugh-Leifson's medium) is selected according to the experimental need and strains of bacteria used. The medium is prepared thicker so as to float the acrylic cast on the surface.

The culture medium is taken to the one-third of the test-tube and the cast is carefully placed bottom down over the medium (Figure 3). In this condition the lower part of the repaired defect will touch the culture medium. The access cavity prepared will now act as the chamber for inoculation. 


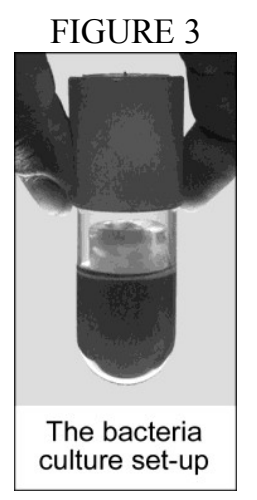

Bacterial strains representing the oral flora may be selected for the test. Generally, $20 \mu 1$ of $1 \times 10^{6} \mathrm{cfu} / \mathrm{ml}$ of the selected strains is used for the bacterial leakage experiments. The access cavity will be sufficient enough to act as a reservoir for the quantity of bacterial inoculum.

Once inoculated, bacteria will always try to migrate to the medium through the path of least resistance, if imperfections are present. In addition, the chemotactic movement of the bacteria towards the nutrient source and the omnipotent gravitation force play roles in the migration of bacteria.

The migration of bacteria to the culture medium could be identified visually when an appreciable volume of colonies are formed in the culture medium. The time taken for this change since the inoculation is recorded.

The PMMA casts of teeth prepared in the same fashion, but without making perforation, could be used as the negative control. Those with through-perforation (i.e. not repaired) will serve as positive control. The test may be repeated several times for a statistically relevant data.

\section{Discussion}

The model for the bacterial leakage studies of perforation repair materials presented here is significantly superior to other models developed for the purpose.

The earliest model used for bacterial penetration studies consists of repaired single root teeth pierced (up to the cemento-enamel junction) through the plastic cap of $12 \mathrm{ml}$ plastic vials containing the culture medium. The access cavity with enlarged coronal portions acted as the upper chamber for inoculation. The joints are sealed with sticky wax and the tooth is covered with layers of nail polish ${ }^{9}$. This model had been followed by others to test root end filling materials ${ }^{10,11,8}$. In a similar model used by Bal et al. ${ }^{12}$, the tooth roots are sealed in the lumen of a latex tubing segment with cyanoacrylate and inserted down into culture medium. More sophisticated models emerged in the past few years. Carratu et al ${ }^{13}$ employed a custom designed glass apparatus for the bacterial penetration study. This is a double tube system having an upper tube carrying the repaired tooth at the end, which is inserted into a bottle having a side-tube intended for the handling of the medium. The sealing is done by silicone paste and cyanoacrylate. A simpler plastic double-tube system could be found in a work by Mangin et al. ${ }^{14}$. Tooth is sealed at the cut end of a $2 \mathrm{ml}$ centrifuge tube and inserted through the cap of a $15 \mathrm{ml}$ plastic tube filled with the medium. The sealing is done with sticky wax.

All the models mentioned above were originally developed for testing the root-end materials filled in single roots. The sealing of the boundaries (using wax, cyanoacrylate and varnish) is done arbitrarily on custom basis, where there are sound chances of imperfections. In order to handle such error factors, a large population of samples are to be tested. More over, these models cannot be applied universally for all kinds of teeth. The use of readily available plastic tubes or custom-blown glass tubes may work for single root teeth, where the size variation is minimal. It is difficult to incorporate molars in the design, owing to their bigger size, non-uniformity in shape, variation in sizes and presence of multiple roots.

In a recent study on the bacterial leakage through perforation repair materials, Ferris and Baumgartner 15 tried to overcome these problems by modifying the design. Here, the upper chamber is a $5 \mathrm{ml}$ syringe (without needle), the tip of which is inserted into the access opening made on the tooth. It is dipped straight into a $20 \mathrm{ml}$ scintillation vial carrying the culture medium. The sealing is done with cyanoacrylate and nail varnish. Though molars are conveniently accommodated in this model, the possible errors due to sealing are still present.

It is notable that the present model avoids all the flimsy and custom sealing, and at the same time, is capable of accommodating tooth of any size and shape. Once the PMMA cast is made, the tooth-acrylic boundaries remain intact.

The block provides a rigid support to the tooth sample and makes it easy to handle during the experiment. When made in compatible with size of the microbiological culture tube, it provides all the advantages of a double-chamber system. 
The sizing of the cast (sectioning out below the furcation level, removing the roots) give the advantage of visibility during the bacterial culture. The onset of migration of bacteria could be identified meticulously, without the obstruction of the roots.

The layer of self-cure clear acrylic provided at the bottom side of the cut section seal any exposed dentinal tubules. It is more impervious and stable than the nail varnish layers and is free from peel-off problems.

The elimination of temperature sensitive sealing materials like sticky wax, cyanoacrylate and nail varnish, makes it possible to sterilize the samples by autoclaving. The need for sophisticated methods like gamma irradiation and ethylene oxide sterilization is avoided here. Autoclaving is the simplest method, which is easily accessible for any microbiology lab.

In vitro investigations of bacterial leakage through endodontic repair materials could be conducted easily and reliably with this model.

\section{References}

[1]. Pashley DH (1990): Clinical considerations of microleakage, J Endodont, 16: 70-77.

[2]. Going RE (1972): Microleakage around dental restorations : A summarizing review, J Amer Dent Asso, 84: $1349-1357$.

[3]. Going RE (1979): Reducing marginal leakage: a review of materials and techniques, J Amer Dent Asso, 99: 646-651.

[4]. Bauer JG, Henson JL (1984): Microleakage- a measure of the performance of direct filling materials, Op Dent, 9: 2-9.

[5]. Browne RM and Tobias RS (1986): Microbial microleakage and pulpal inflammation - A review, Endodont Dent Traumatol, 2: 177-183.

[6]. Wu MK, Wesselink PR (1993): Endodontic leakage studies reconsidered- Part I. Methodology, application and relevance, Int Endod J, 26: 37-43.

[7]. Goldman M, Simmonds S, Rush R (1989): The usefulness of dye penetration studies re-examined, Oral Surg Oral Med Oral Path , 67: $327-332$

[8]. Torabinejad M, Rastegar AF, Kettering JR, Pitt Ford TR (1995): Bacterial leakage of Mineral Trioxide Aggregate as a root end filling material, J Endodont, 21: 109-112.

[9]. Kos WL, Aulozzi D P and Gerstein H (1982): A comparative bacterial microleakage study of retrofilling materials, J Endodon, 8: 355-358.

[10]. Torabinejad M, Ung B, Kettering JD (1990): In vitro bacterial penetration of coronally unsealed endodontically treated teeth, J Endod, 16: 566-569.

[11]. 11.Fischer EJ, Arens DE, Miller CH (1998): Bacterial leakage of mineral trioxide aggregate as compared with zinc-free Amalgam, Intermediate Restorative Material and Super-EBA as a root-end filling material, J Endodont, 24: 176-179.

[12]. Bal AS, Hicks ML, Barnett F (2001): Comparison of laterally condensed .06 and .02 tapered Gutta-percha and sealer in vitro, J Endodon, 27: 786-788.

[13]. Carratu P, Amato M, Riccitiello F, Rengo S (2002): Evaluation of leakage of bacteria and endotoxins in teeth treated endodontically by two different techniques, J Endodon, 28: 272-275.

[14]. Mangin C, Yesilsoy C, Nissan R, Stevens R (2003): The comparative sealing ability of hydroxyapatite cement, Mineral Trioxide Aggregate, and Super Ethoxybenzoic Acid as root-end filling materials, J Endodon, 29: 261-264.

[15]. Ferris DM, Baumgartner JC (2004): Perforation repair comparing two types of Mineral Trioxide Aggregate, J Endodon, 30: 422424. 